Rev SINAPSIS, Vol. 8, Nº 1, Junio 2016

ISSN $1390-7832$

\title{
Manual de Procedimientos Administrativos de la Empresa Apronam S.A. del Cantón Chone
}

\section{Manual de Procedimientos Administrativos}

$\mathrm{L}$

Lic. Rosa Yesenia Vera Loor, Mgs. c.a. ${ }^{(1)}$

Dr. Zarcos Zulio Zambrano Zambrano ${ }^{(2)}$

Eco. Tania Garcia Ponce, Mgs. c.a. (3)

(1) Universidad Estatal del Sur de Manabí, Jipijapa, Ecuador

(2) Universidad Laica Eloy Alfaro de Manabí, Chone, Ecuador

(3) Universidad Estatal del Sur de Manabí, Jipijapa, Ecuador

Contacto: yeseniavera04@hotmail.com

Receptado: 24/03/2016 Aceptado: 14/05/2016

\section{Resumen}

El presente trabajo fue realizado en la Empresa APRONAM S.A. del Cantón Chone, donde se detectó un deficiente proceso de gestión administrativa y financiera. Se desarrolló una investigación de campo, modalidad bibliográfica y cuasi experimental, teniendo como objetivo: Elaborar un manual de procedimientos de Gestión Administrativa y Financiera de la empresa APRONAM S.A. del Cantón Chone, determinándose que no existen un manual de procedimientos definido para realizar los procesos de gestión administrativa y financiera, por lo que se propone elaborar un manual de procedimientos de gestión administrativa y financiera para optimizar estos procesos, para ello se han aplicado, leyes, reglamentos, estatutos y disposiciones emanadas por los organismos de control como son el Servicio de Rentas Internas, la Superintendencia de Bancos, Superintendencia de Compañías, aplicando las Normas Ecuatorianas de Contabilidad y Normas Ecuatorianas de Auditoria, para que la empresa cuente con flujogramas que faciliten conocer y seguir los procesos para optimizar los procedimientos con la intención de que las actividades que se realizan día a día las ejecute el personal con eficiencia y efectividad optimizando tiempo y recursos.

Palabras claves: Manual de procedimientos administrativo, financiero, contable, flujogramas 
Rev SINAPSIS, Vol. 8, Nº 1, Junio 2016

ISSN 1390 - 7832

\section{Manual of procedures of administrative and financial management of the company APRONAM S.A. of the Canton Chone, Ecuador}

The present work was carried out at the company APRONAM S.A. Canton Chone, where was detected a poor administrative and financial management process. It was developed a bibliographic and quasiexperimental research field. The aim of this research was: To develop a manual of procedures of administrative management and financial of the company APRONAM S.A. in the Canton Chone, so that there is not a manual of procedures defined to perform administrative and financial management processes, therefore intends to develop a manual of procedures for managing administrative and financial to optimize these processes for that reason it was applied, laws, regulations, statutes and provisions emanated by the control agencies such as the Internal Revenue Service, the Superintendence of Banks, Superintendence of Companies, Ecuadorian Accounting Standards, Ecuadorian Standards of Financial Audit so the company has flowcharts that facilitate to know and follow the processes to optimize procedures with the intention that staff activities performed daily in the company are implemented efficiently and effectively which allow to optimize time and resources.

Keywords: Manual of procedures, administrative, financial, accounting, flowcharts

\section{Introducción}

Conforme avanzan las empresas se hace necesaria la creación de "Manuales" sobre la actuación individual o por funciones de los trabajos dentro de ellas, cada día es más evidente esta necesidad; ya que es la única forma de establecer parámetros de acción operativa de los empleados, y por ende, agilizar la puesta en marcha de los objetivos que se quieran lograr.

Un manual es un conjunto de documentos que partiendo de los objetivos fijados y las políticas implantadas para lograrlo, señala la secuencia lógica y cronológica de una serie de actividades, traducidas a un procedimiento determinado, indicando quien los realizará, que actividades han de desempeñarse y la justificación de todas y cada una de ellas, en forma tal que constituyen una guía para el personal que ha de realizarlas (Gómez, 1994).

Es un instrumento de información en el que se consignan metódicamente las operaciones que deben seguirse para la realización de las funciones de una o varias entidades (Barrera, 2005) 
La gestión hace referencia a la acción y a la consecuencia de administrar o gestionar algo. Montiel, Kadi 2012 Vol. 4. La gestión por procesos hace compatibles las necesidades organizativas internas con la satisfacción de los clientes. (Pérez, 2010).

Los procedimientos consisten en describir detalladamente cada una de las actividades a seguir en un proceso laboral, por medio del cual se garantiza la disminución de errores (Melinkoff, 1990).

La elaboración de instructivos de procedimientos, o sea el planteamiento por escrito de la forma en que debe realizarse cada trámite. Todos los instructivos de procedimiento de la institución, o de un área concreta de esta se unen para formar lo que se conoce como manual (Kramis, 1994 ), en un manual quedan ilustradas los procedimientos que realiza la institución en cada área de trabajo.

Los diagramas de flujo son una parte importante del desarrollo de procedimientos, debido a que por su sencillez gráfica permite ahorrar muchas explicaciones. (Álvarez. 1996)

Para lograr la correcta administración de una empresa es imprescindible contar con un adecuado sistema de información que permita tomar decisiones acertadas y en el momento preciso. De este sistema de información integral forma parte el sistema contable, que tiene como objetivo la capacitación y procesamiento de datos que permitan alcanzar los objetivos y fines de la contabilidad (Vázquez \& Bongianino, 2008).

Por tanto se considera que el Manual de Procedimiento es un documento de gestión que describe en forma pormenorizada y secuencial las operaciones que se deben seguir en cada proceso para la ejecución de los procedimientos en un área o departamento de la Empresa.

\section{Materiales y métodos.}

En los métodos y técnicas que se emplearon están: Investigación descriptiva y métodos empíricos, a través de los cuales se buscó información que brinda la posibilidad de hacer valoraciones y juicios de profundidad acerca de los mismos, por otra parte se emplearon métodos de ordenamiento y organización de los datos para complementar lo cualitativo y cuantitativo.

Se empleó la técnica de observación directa, entrevista y encuestas: Con lo que se determinó que no existe un Manual de Procedimientos de Gestión Administrativa y Financiera. 
Además se utilizó el análisis reflexivo para establecer relaciones que existen entre las diferentes concepciones de los diferentes autores; y, la inducción y deducción para poder partir de lo general a lo específico para la aplicación de este manual adecuado a la empresa, lo mismo que se valoró por criterio de expertos y reflexiones colectivas.

\section{Resultados}

La existencia de un manual de procedimientos sirve para que la administración aumente la posibilidad de que los empleados utilicen los sistemas y procedimientos establecidos al llevar a cabo sus tareas.

Gráfico No 1: Diagrama de Flujo de Procesos Administrativos y Financieros claves

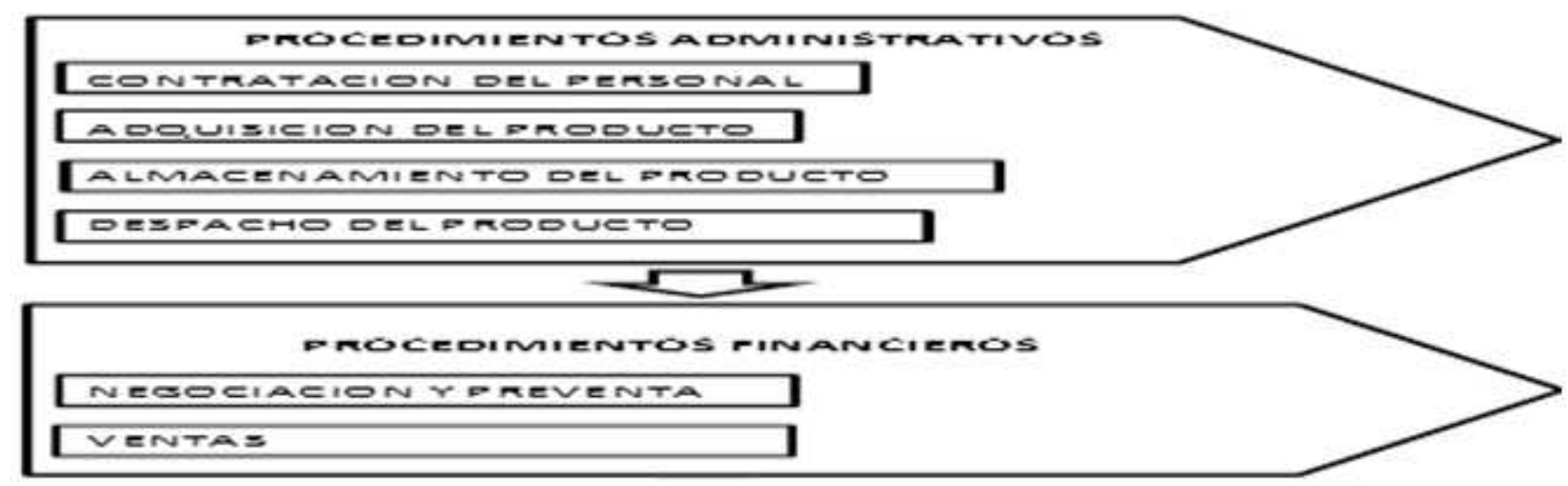

\section{Procedimientos Administrativos}

Dentro de los procedimientos administrativos de la empresa se determinó que son cuatro los fundamentales y están descritos en los gráficos 2,3 y 4.

\section{Descripción de procedimientos:}

\section{Contratación del personal}

1. El departamento solicitante envía el requerimiento de personal al Gerente General el cual analiza el requerimiento de personal, aprueba el requerimiento.

2. Gerente General se reúne con el asistente administrativo y define la remuneración correspondiente al puesto vacante. 
3. El asistente administrativo prepara la entrevista inicial y elabora la prueba de conocimientos a rendir los aspirantes y publica en prensa local el perfil requerido y los beneficios ofrecidos por la empresa después convoca a los aspirantes a la entrevista inicial y a rendir la prueba de conocimientos.

4. Gerente General Analiza las entrevistas realizadas y califica las pruebas de conocimientos aplicadas y a la persona elegida.

5. El asistente administrativo elabora e imprime el aviso de entrada al IESS de la persona contratada y lo envía al gerente general. Además elabora 3 ejemplares del contrato de trabajo de la persona contratada y los envía al gerente general.

6. Gerente General Firma el aviso de entrada al IESS y los 3 ejemplares del contrato de trabajo junto a la persona contratada.

7. El asistente administrativo Legaliza el contrato de trabajo en el Ministerio de Relaciones Laborales. Archiva el contrato de trabajo legalizado, el aviso de entrada al IESS, la hoja de vida, el requerimiento de personal, la guía de entrevista inicial y la prueba de conocimientos y entrega a la persona contratada la lista de los documentos que debe presentar para realizar su ingreso formal a la empresa y comenzar con la capacitación para el puesto de trabajo aprobado.

\section{Adquisición del producto}

1. Para realizar los requerimientos se reúne el bodeguero, junto con control operativo (Facturador)

2. Reciben solicitudes directas de la fuerza de ventas, y el personal administrativo donde ello solicitan requerimientos extras registrando en el formato de requerimientos que deben ser tomados en cuanta en el momento que se haga el pedido a nuestro proveedor.

3. En el momento que reciben las solicitudes mediante el formato de requerimientos por parte de la fuerza de venta y personal administrativo, el bodeguero y control operativo llenan su registro con todos los pedidos a realizarse según el día a realizarse debido a que se hace pedidos de diferentes productos en diferentes días.

4. Supervisor de ventas revisa, analiza los requerimientos que va a realizarse por parte del control operativo. 
5. Si es que no aprueba los requerimientos, control operativo realiza los cambios necesarios y procede a realizar el pedido. De lo contrario si es que no se realizan cambios en los requerimientos control operativo realiza el pedido directamente.

Gráfico No. 2: Flujograma del procedimiento de adquisición del producto

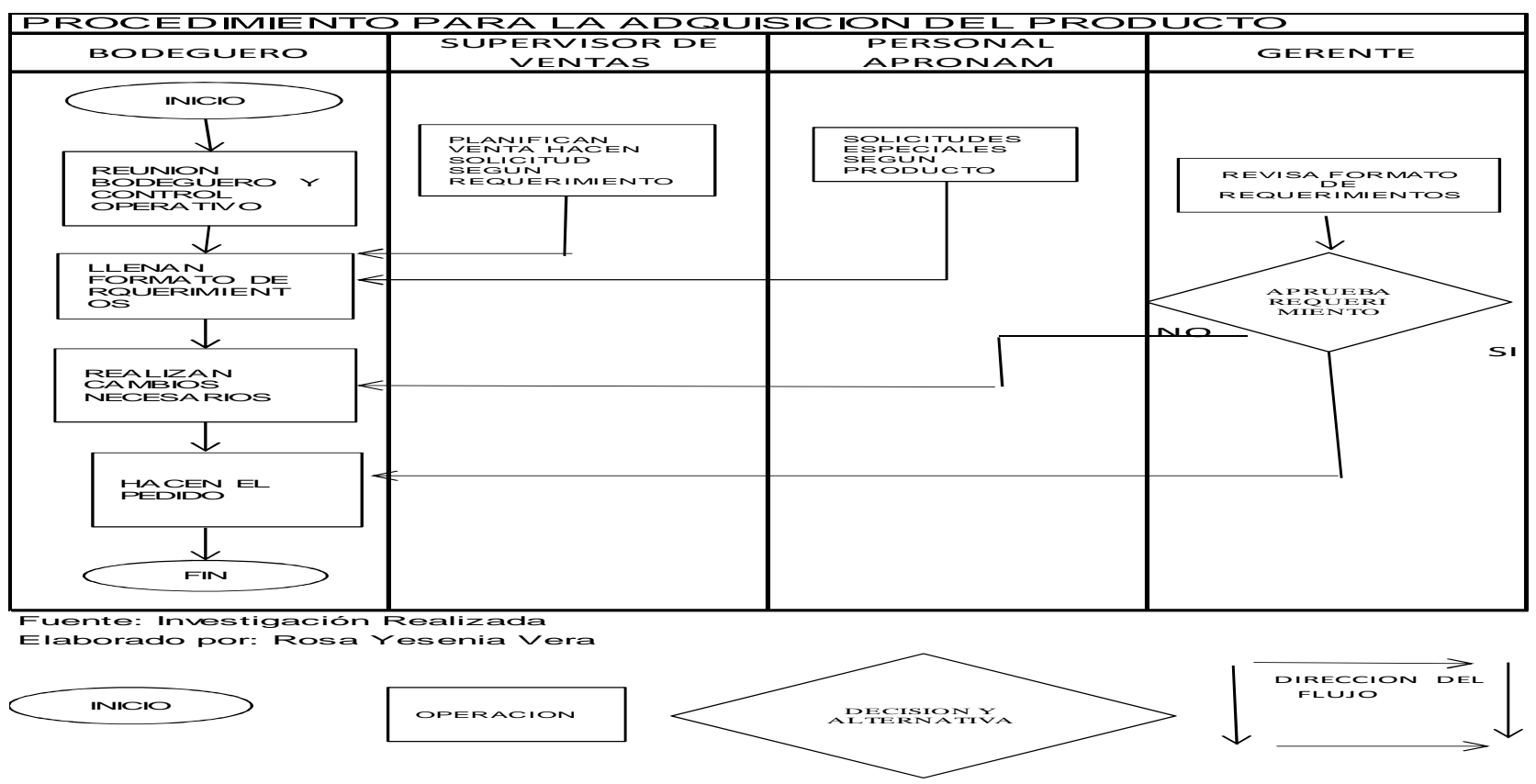

\section{Almacenamiento del producto}

1. El proveedor hace la entrega del producto que se había hecho el requerimiento en el proceso de adquisición del producto.

2. El bodeguero recibe los productos por parte de nuestro proveedor en cuanto a frescos, congelados, embutidos y secos se refiere.

3. El ayudante de bodega pasa una por una las gavetas que contienen los productos detallados en el punto anterior. Mientras el ayudante de bodega pesa y dicta el peso el bodeguero compara con las facturas si el peso facturado es igual al real.

4. El ayudante de bodega una vez realizada la comparación de pesos empieza a estibar en cada una de las cámaras el producto recibido de acuerdo a la fecha de elaboración o a bodega si es producto seco. 
Rev SINAPSIS, Vol. 8, No 1, Junio 2016

ISSN $1390-7832$

5. Estiba el producto recibido.

6. Al recibir todos los productos frescos, congelados, embutidos y secos y constata que todo estuvo conforme se firma la conformidad del cliente.

7. Facturación empieza a ingresar las compras al sistema para poder empezar con el proceso de negociación y preventa.

Gráfico No. 3: Flujograma del procedimiento de almacenamiento del producto

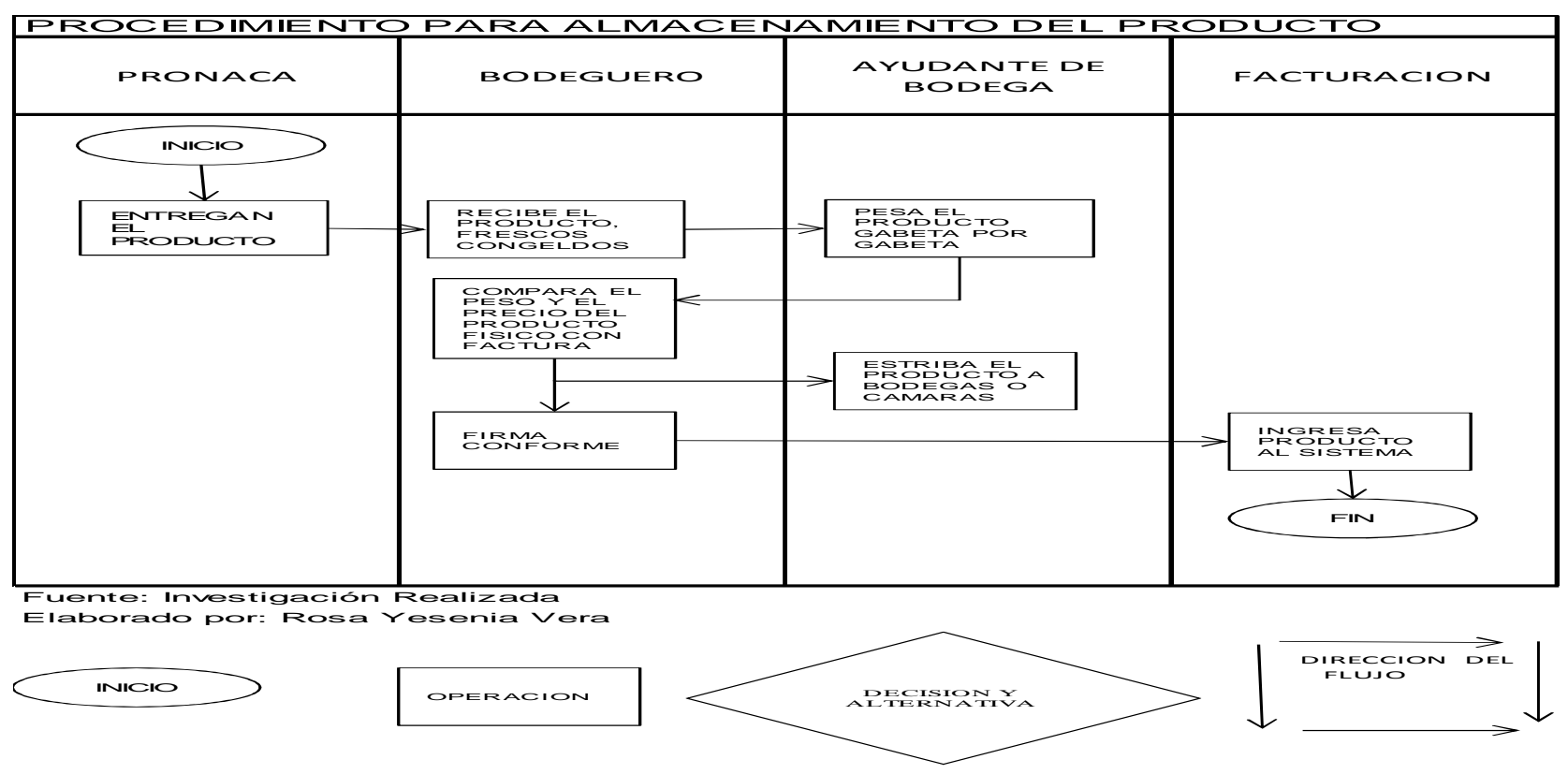

\section{Despacho del producto}

1. Con la información recibida de preventa comienzan el proceso de despacho.

2. Facturación baja esa información y empieza a sacar cada una de la guía de remisión, los consolidados de los camiones y las facturas de cada uno de ellos.

3. Los bodegueros reciben los consolidados, y empiezan a clasificar y según el horario de salida de los camiones continúan con el proceso.

4. El ayudante de bodega pesa los productos y cuenta las unidades, las clasifican por clientes a atender. 
Rev SINAPSIS, Vol. 8, Nº 1, Junio 2016

ISSN $1390-7832$

5. Los ruteros y choferes empiezan a estibar el camión según el orden de entrega de cada uno de los clientes, para empezar con el proceso de venta.

Gráfico No. 4: Flujograma del procedimiento de despacho del producto

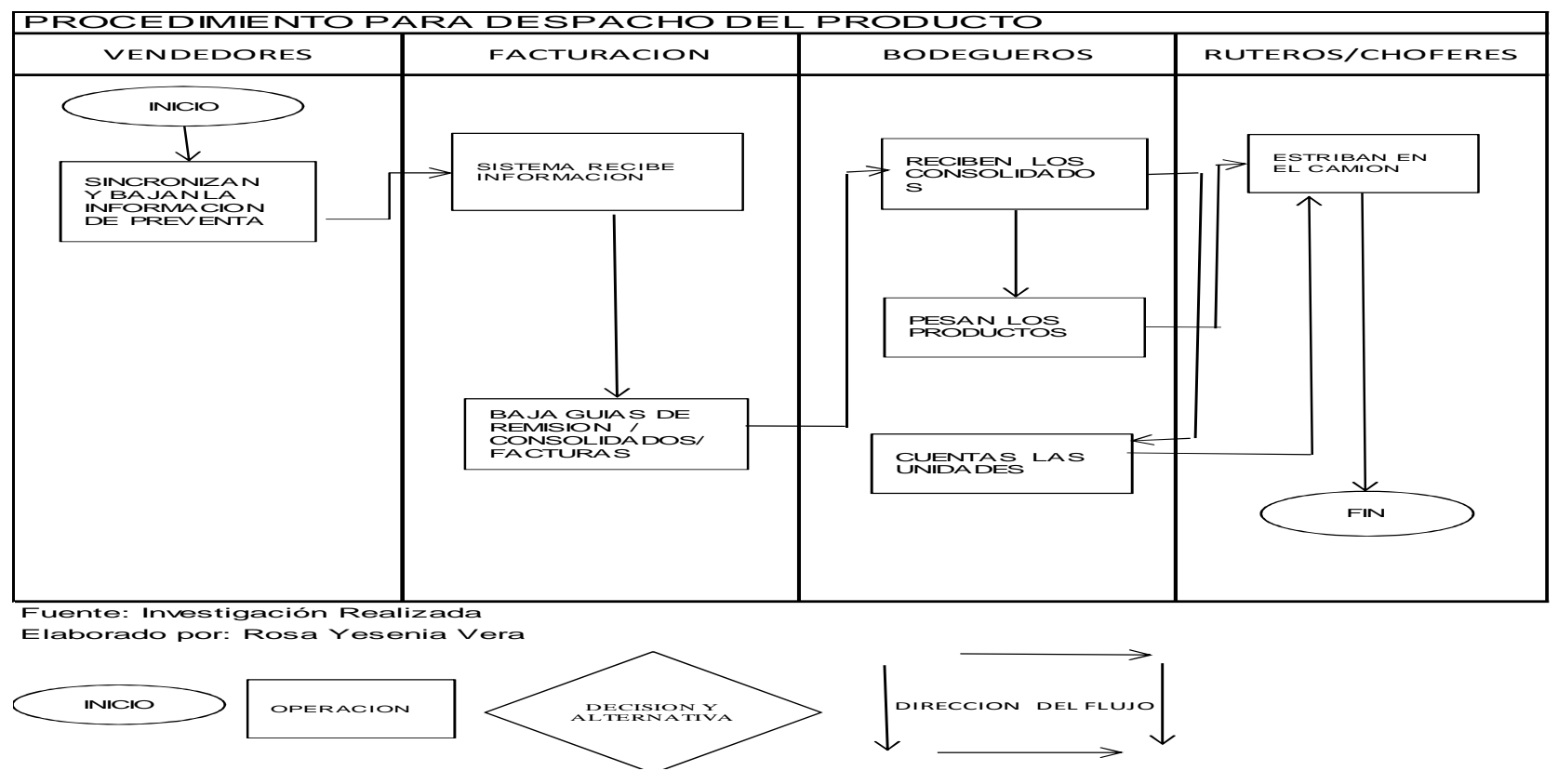

\section{Procedimientos Financieros:}

Los procedimientos financieros considerados básicos para el desarrollo empresarial de esta compañía son los descritos en el gráfico 4.

\section{Negociación y preventa}

1. Los vendedores revisan en su PDA cada uno de los clientes que tiene que visitar ese día, y sincronizan la información donde detalla lo que tienen en inventario para poder negociar y prevender.

2. Al llegar donde el cliente saluda y le presenta las carteras pendientes de pago, si es que el cliente no paga la factura pendiente no toma el pedido, y si el cliente paga la factura vencida el vendedor procede a continuar con el proceso.

3. El vendedor aplica las estrategias de venta establecidas para este proceso, y logra que el cliente realice el pedido en mayor cantidad posible e ingresa este pedido en su PDA. 
Rev SINAPSIS, Vol. 8, Nº 1, Junio 2016

ISSN 1390 - 7832

4. Después de recorrer todos los clientes de la ruta y aplicar el mismo procedimiento regresan a la empresa y sincronizan todos los pedidos, pagos que se obtuvieron ese día.

5. La facturadora recibe toda esa información para proceder a continuar con el despacho del producto.

\section{Ventas}

1. Al estibar el camión de acuerdo al orden de entrega de clientes el rutero firma la guía de remisión y recibe las facturas.

2. Empiezan a recorrer uno a uno los clientes y entrega el producto que le corresponde a cada uno de ellos, si el cliente es con crédito, ellos dejan el producto y se retiran.

3. Si el cliente es a contado el rutero cobra la factura correspondiente, y si el cliente paga la factura el proceso llega a su fin. Si el cliente no paga la factura el rutero y el chofer empiezan a retirar el producto, y lo embarcan nuevamente en el camión.

4. Al regresar el camión después de todas las entregas, llegan a la distribuidora y bajan todos los productos que han retornado, producto que es recibido y contado por el Jefe de Bodega

Gráfico No. 4: Flujograma del procedimiento de ventas

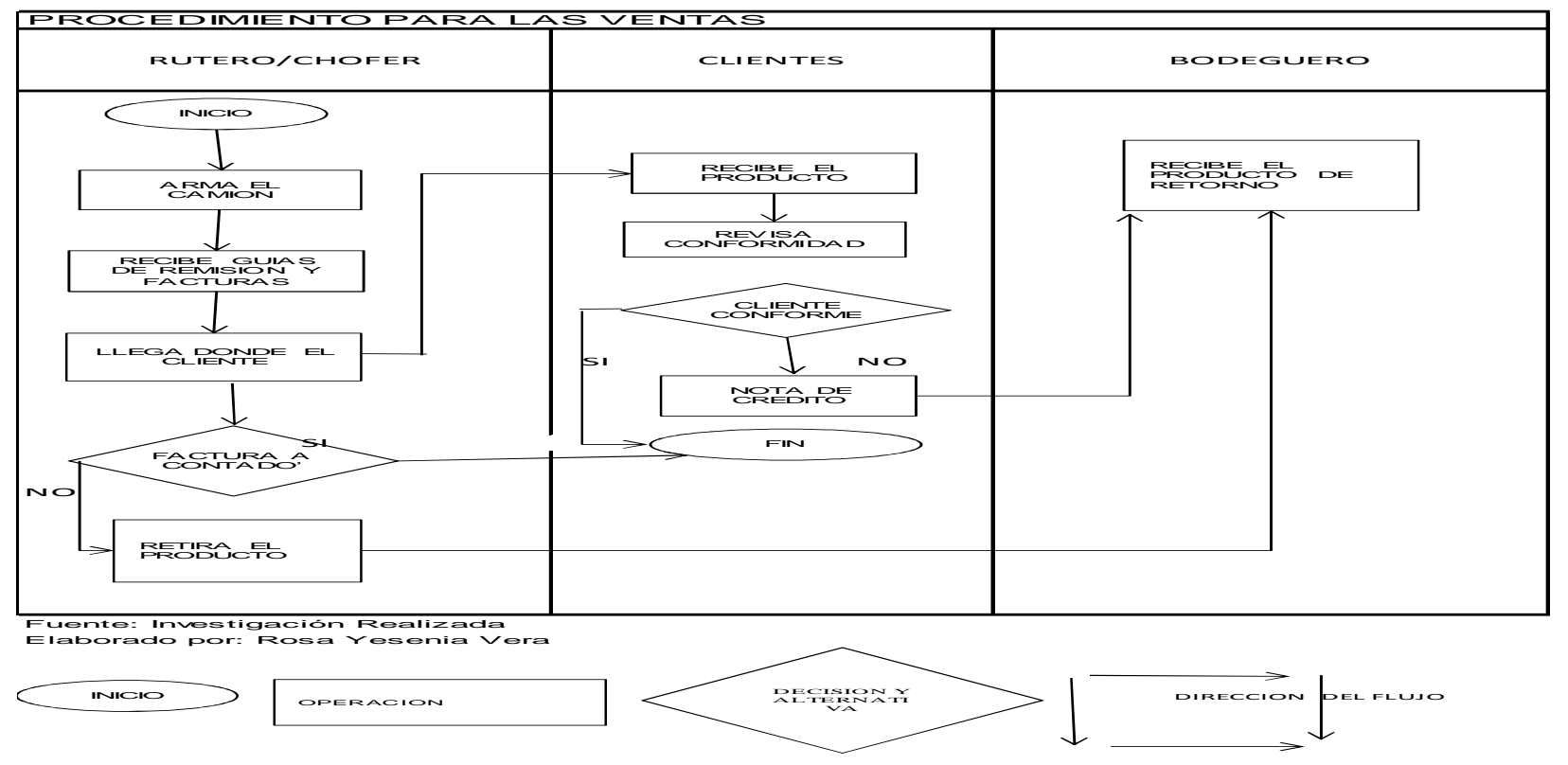




\section{Discusión}

Los Manuales en la actualidad son herramientas de gran importancia para las empresas, ya que gracias a su flexibilidad les permiten asumir cambios en el mundo empresarial lo que permite lograr los objetivos institucionales de manera más organizada.

Los procedimientos son planes que establecen un método para manejar las actividades futuras" convirtiéndose en guías de acción que detallan la forma bajo la cual ciertas actividades deben cumplirse. Procedimientos es el conjunto o proceso de pasos, ampliamente vinculados y ordenadamente ubicados, realizados y dirigidos a precisar la forma de hacer algo, incluyendo el qué, cómo y a quién incumbe el desarrollo de la tarea (Koontz, 1996).

Los procedimientos generan diversos beneficios como: Ayudan a imponer un nivel de consistencia en toda la organización Facilitan la delegación de autoridad y la fijación de responsabilidad Conducen al desarrollo de métodos más eficientes de operación facilitan la acción de controlar Ayuda a lograr coordinación de actividades Conservan el esfuerzo administrativo permitiendo economías de personal (Rodríguez, 2002).

Los procedimientos administrativos facilitan la planeación de la administración y el control de las operaciones (Horngren. Sundem \& Elliott, 2000). Los procedimientos administrativos constituyen un factor decisivo que afecta a la calidad del servicio prestado. Dotar de agilidad y rapidez a los procedimientos tiene una repercusión directa en la satisfacción del cliente. (Heredero, 2004).

Los procedimientos financieros forman parte de la contabilidad, por lo que esta se constituye como una herramienta indispensable para la toma de decisiones, por lo que la información que genera debe propender a: Coordinar las actividades económicas y administrativas. Captar, medir, planear y controlar las operaciones diarias. (Meigs, 2000).

El empresario por medio de la contabilidad tendrá constancia de la situación de su patrimonio y controlara su evolución dinámica patrimonial con un ordenado registro de datos, cuyo objetivo es informar de la situación de la empresa (Omeñaca, 2008).

La contabilidad proporciona datos, para convertirlos en indicadores de actuación, en cierto modo coadyuvan a evaluar la trayectoria de la organización, da un parámetro general del valor de la misma en 
el tiempo (Morgado, 2010). La información contable es útil para cualquiera que deba emitir juicios y tomar decisiones que generen consecuencias económicas (Sánchez, 2002).

Con la aplicación de este instrumento la empresa contara con una secuencia gráfica de los procesos y procedimientos a aplicar para lograr conseguir las metas propuestas en las actividades propias de la entidad, con la finalidad de dar una atención rápida y oportuna al cliente cuando desea adquirir el producto que se comercializa, logrando la satisfacción del mismo y la ejecución del trabajo por parte del empleado en el menor tiempo posible.

\section{Conclusiones}

Se identificó que la gestión tanto en términos administrativos, financieros y contables ha evolucionado de manera significativa desde sus inicios hasta la actualidad, llegando hacer hoy por día un proceso que logra que las personas involucradas cumplan con eficiencia los objetivos trazados.

Los diferentes aspectos teóricos comprueban que tanto la gestión administrativa y financiera se basan en la consecución eficaz de las actividades, orientadas a coordinar los recursos disponibles para conseguir los objetivos de la empresa en el menor tiempo posible. 
Rev SINAPSIS, Vol. 8, No 1, Junio 2016

ISSN $1390-7832$

\section{Bibliografía}

1. Álvarez, M G (1996) Manual para elaborar Manuales de Políticas y Procedimientos. México D.F.: Panorama

2. Barrera, A (2005) Recuperado de ri.ufg.edu.sv/jspui/bitstream/11592/.../657.458M722m-Capitulo\%20II.pdf

3. Gómez, G. Planeación y Organización de Empresas. Recuperado de https://administraciondepersonal.files.wordpress.com/2009/08/planeacion-yorganizacion-de-empresas-capitulo-7.pdf

4. Heredero, C (2004) Ilustraciones de la aplicación de las tecnologías de información en la empresa Española. Madrid, España: Esic

5. Horngren, Ch. Sundem, G. \& Elliot, J (2000) Introducción a la Contabilidad Financiera. México: Pearson Educación

6. Koontz, H. (1996) Administración una perspectiva global. México: McGraw Hill.

7. Kramis, J L (1994) Sistemas y procedimientos administrativos: Metodologia para su aplicación en instituciones privadas y públicas. México: Universidad Iberoamericana. A.C.

8. Meigs (2000). Recuperado de repositorio.ute.edu.ec/bitstream/123456789/4296/1/58307_1.pdf

9. Melinkoff, R. (1990) Los Procesos Administrativos. Caracas, Venezuela: Panapo.

10. Montiel, Kadi 2012 Vol. 4, No.1, Pensamiento estratégico para la gestión pública: una confluencia sobre la planificación, organización, dirección y control. Documento en línea. Disponible en http://www.publicaciones.urbe.edu/index.php/coeptum/article/view/

Manual de Procedimientos Administrativos 
Rev SINAPSIS, Vol. 8, No 1, Junio 2016

11. Morgado (2010) Recuperado de http://docplayer.es/2277187-Lic-juana-mmorgado-c-contabilidad-i-enero-2-010.html

12. Omeñaca (2008). Recuperado de http://repo.uta.edu.ec/bitstream/123456789/1931/1/TA0194.pdf

13. Pérez, J A (2010) Gestión por procesos. Madrid, España: Esic

14. Rodríguez, J. Cómo elaborar y usar los manuales administrativos. Recuperado de https://issuu.com/cengagelatam/docs/como_elaborar_manuales_administrativos_r odriguez_v/4

15. Vázquez, R y Bongianino, C. (2008) Principios de la teoría contable. Buenos Aires, Argentina: Aplicación Tributaria S.A.

Manual de Procedimientos Administrativos 